\title{
Study on the Influence of Electromagnetic Pulse on UAV Communication Link
}

\author{
Yinsen Jia', Xinqi Tu', Wei Yan,2,*, Enrong Wang ${ }^{1}$, Yang Zhao', Shuqing Ding ${ }^{3}$ \\ ${ }^{1}$ School of Electric and Automation Engineering Nanjing Normal University, Nanjing, Jiangsu 210042, China \\ ${ }^{2}$ UAV Research Institute Nanjing Aeronautics and Astronautics University, Nanjing, Jiangsu 210016, China \\ ${ }^{3}$ Nanjing Special Equipment Inspection Institute, Nanjing, 210002, China \\ *Corresponding author: 61197@njnu.edu.cn
}

Received June 12, 2019; Revised July 15, 2019; Accepted July 26, 2019

\begin{abstract}
UAVs have many functions in detection, fire protection, communication, etc. in modern life, but the complex electromagnetic environment has a very bad impact on the UAV communication link. In this paper, the BPSK modulation and demodulation method of the UAV downlink communication link was analysed. Some typical electromagnetic pulse models were established, including sinusoidal pulse, square wave pulse, triangular wave pulse, Gaussian noise, electric fast transient pulse and surge pulse. The amplitude of different electromagnetic pulses was analysed when UAV communication error rate achieve 5\%. Furthermore, the influence on the transmission image quality via UAV downlink was analysed with different electromagnetic pulses, which will provide a theoretical basis for the anti-interference optimization design of UAV communication links in the future.
\end{abstract}

Keywords: UAV, communication link, electromagnetic pulse, BPSK, bit error rate

Cite This Article: Yinsen Jia, Xinqi Tu, Wei Yan, Enrong Wang, Yang Zhao, and Shuqing Ding, "Study on the Influence of Electromagnetic Pulse on UAV Communication Link." American Journal of Electrical and Electronic Engineering, vol. 7, no. 2 (2019): 42-48. doi: 10.12691/ajeee-7-2-4.

\section{Introduction}

Since the 20th century, with the maturity of communication technology, aviation technology, and electronic technology, the efficiency, distance, and accuracy of unmanned aerial vehicle (UAV) communication are asked for higher requirements. UAVs are often used for important inspection tasks such as industrial facility inspection, nuclear pollution detection, building inspection, firefighting, military investigation, and long-range attacks. UAVs are already essential in daily life and in the military $[1,2,3]$. At present, the UAV communication link is regarded as the main research object by many researchers $[4,5]$. However, in actual operation, great challenges are posed to the safe operation and information transmission of UAV because of the wide application of high-power microwave, electromagnetic pulse, fire control radar and high-frequency electronic equipment. Complex electromagnetic environment includes strong electromagnetic pulse such as sinusoidal pulse, square wave pulse, triangular wave pulse, Gaussian noise, electric fast Transient pulse groups (EFT), surge pulses etc. In 2011, the US military RQ-170 sentinel stealth UAV was captured by Iran based on electromagnetic interference device. In 2014, the US MQ-9 UAV lost communication with the ground control station shortly after taking off from the secret base in the Middle East. In 2015, the US MQ-9 UAV crashed near the Bagram airport in Afghanistan. In 2015, the US Global Hawk UAV was subjected to radio interference over the Nansha Islands, causing distortion of the transmission picture. In 2016, the Japanese detective UAV disappeared in the air near Fukui Prefecture.

Recently, to the electromagnetic environment interference problem of UAVs, the transient voltage and current of various cables under electromagnetic pulse radiation are analysed, and a more reasonable UAV cable layout scheme was proposed [6]. The Electromagnetic Compatibility Simulation Model of Composite UAV has been established by the method of Finite time domain differential electromagnetic field [7]. The impulse noise interference model of the marine UAV communication link has been established [8]. An analysis method for BER performance of power line communication systems considering the effects of impulse noise and background Gaussian noise was proposed [9]. A performance test method for UAV communication links in extreme environments was presented [10]. The ultra-wideband electromagnetic pulse radiation system has been used to conduct the irradiation effect experiment on the UAV communication link, illustrating that the UAV communication link lost lock is related to the irradiation field strength and the trigger frequency, and the demodulation signal, the bit error rate increases with the decrease of signal-to-noise ratio [11]. The influence of airborne electromagnetic interference on the UAV formation communication link has analysed [12]. Some typical electromagnetic interference (EMI) with their impact and solution has been analysed [13]. Audible noise, and radio interference have been analysed by using Physical models and linear fitting methods [14]. the 
wireless channel model of MATLAB/Simulink has been used to analyse the problem of electromagnetic interference between UAV and ultra wide band (UWB) system [15]. The radiated EMI noise analysis can be simplified by the approach of fast diagnosis, prediction and signal characterization [16]. A noise separation method for conducting EMI based on FPGA has been proposed [17].

However, in the above research, most of the noise analysis of the UAV communication link uses single noise as the analysis target, and in today's UAV signal transmission, many typical noises have not been studied. Meanwhile, the past research the analytic function of the demodulation signal and electromagnetic interference of the communication link has not been established. Therefore, it is difficult to quantitatively calculate the influence of the interference source characteristics or the interference transmission mode or the interference application continuity on the error rate of the demodulated signal, which also brings a lot of obstacles to suppression methods. Accordingly, a typical electromagnetic pulse model including sinusoidal pulse, square wave pulse, triangular wave pulse, Gaussian noise, electrical fast transient pulse group (EFT) and surge pulse is established, and the modulation and demodulation principle of the UAV communication link has been analysed in this paper. The analysis of the amplitude of different electromagnetic pulses at 5\% error rate and its influence on the image transmission quality of UAVs provides a theoretical basis for the anti-interference optimization design of UAV communication links in the future.

\section{BPSK Modulation and Demodulation Principle of UAV Communication Link}

Phase Shift Keying (PSK) modulation and demodulation methods are widely used in modern UAV communication. BPSK and QPSK devices are simple and anti-noise. 8PSK and 16PSK have high spectrum utilization and are good at transmission, but their signal is weaker and their noise immunity is weak [18]. At the same time, since the principle of PSK modulation and demodulation is basically similar, the BPSK modulation and demodulation principle with the best anti-noise performance and strong adaptability to nonlinear channels and fading channels [19] is analysed in this section.

The BPSK modulation process is shown in Figure 1(a). First, the input binary code $S(t)$ is converted into a square wave signal $S_{1}(t)$ having an amplitude of \pm 1 by the pattern conversion module.

$$
S_{1}(t)=2 S(t)-1
$$

Where $t$ is time. The signal $S_{1}(t)$ is multiplied by the carrier signal $\cos \left(f_{c} t+\varphi\right)$ to obtain a BPSK modulated signal $S_{2}(t)$, where $f_{c}$ is the carrier frequency and $\varphi$ is the initial phase angle.

$$
S_{2}(t)=S_{1}(t) \cos \left(2 \pi f_{C} t+\varphi\right) .
$$

The BPSK demodulation process is as shown in Figure 1(b). First, the modulation signal $S_{2}(t)$ is multiplied by the carrier signal $\cos \left(f_{c} t+\varphi\right)$ to obtain a multiplication signal $S_{3}(t)$.

$$
S_{3}(t)=S_{2}(t) \cos \left(2 \pi f_{C} t+\varphi\right)
$$

After passing through the low pass filter, a demodulation signal $S_{4}(t)$ is obtained as a sampling decision.

$$
s_{4}(t)= \begin{cases}0, & s_{3}(t) \leq 0 \\ 1, & s_{3}(t) \geq 0\end{cases}
$$

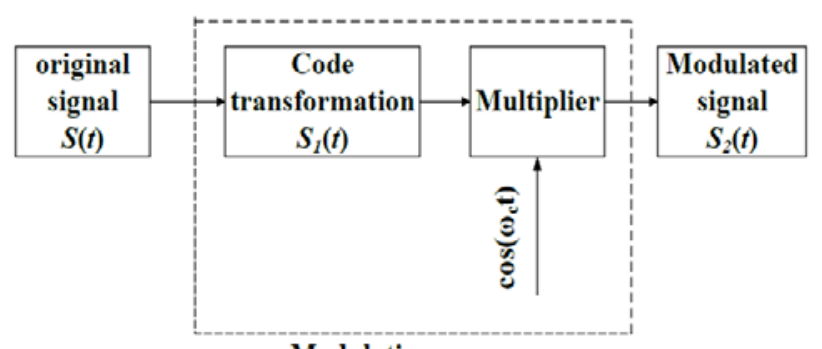

Modulation process

a

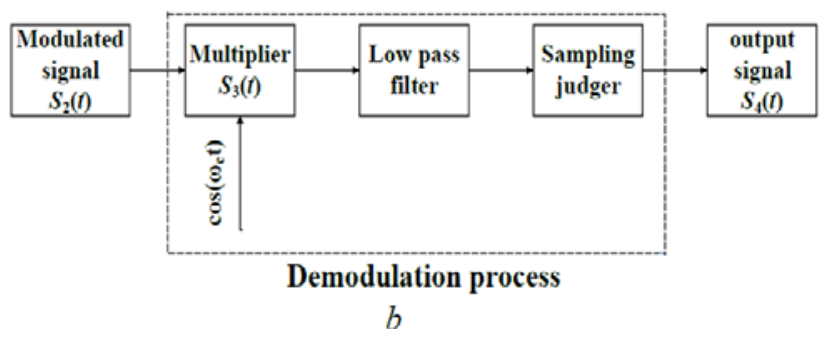

Figure 1. BPSK modulation and demodulation: (a) BPSK modulation process, (b) BPSK demodulation process

\section{Influence of Electromagnetic Interference on Communication Links}

This section mainly analyses the UAV downlink transmission process and establishes a UAV communication link model. Using the established typical pulse model for simulation, and using image data as the original data, after the process of modulation, noise addition, demodulation and outputting the image, the amplitude of different electromagnetic pulses at 5\% error rate and their impact on image transmission quality is analysed through the change of the image.

The block diagram of the downlink transmission process is showed in Figure 2. The UAV collects the data as the original signal $S(t)$. After the BPSK modulation process, the modulated signal $S_{2}(t)$ is obtained. Then the antenna generates the transmitted signal electric field $E_{R}(x, y, z)$, which is the electric field intensity of the transmitted signal at the space $(x, y, z)$. The electric field of the electromagnetic (EM) pulse is $E_{P}$, and $E_{P}(x, y, z)$ is the electric field intensity of the EM pulse at the space $(x, y, z)$. Based on the principle of electromagnetic field vector superposition, the total electric field intensity can be obtained as

$$
\vec{E}(x, y, z)=\overrightarrow{E_{P}}(x, y, z)+\overrightarrow{E_{R}}(x, y, z) .
$$

After superimposing the electric field $E$, the demodulated signal $S_{4}(t)$ is obtained through the antenna and BPSK demodulation process. 


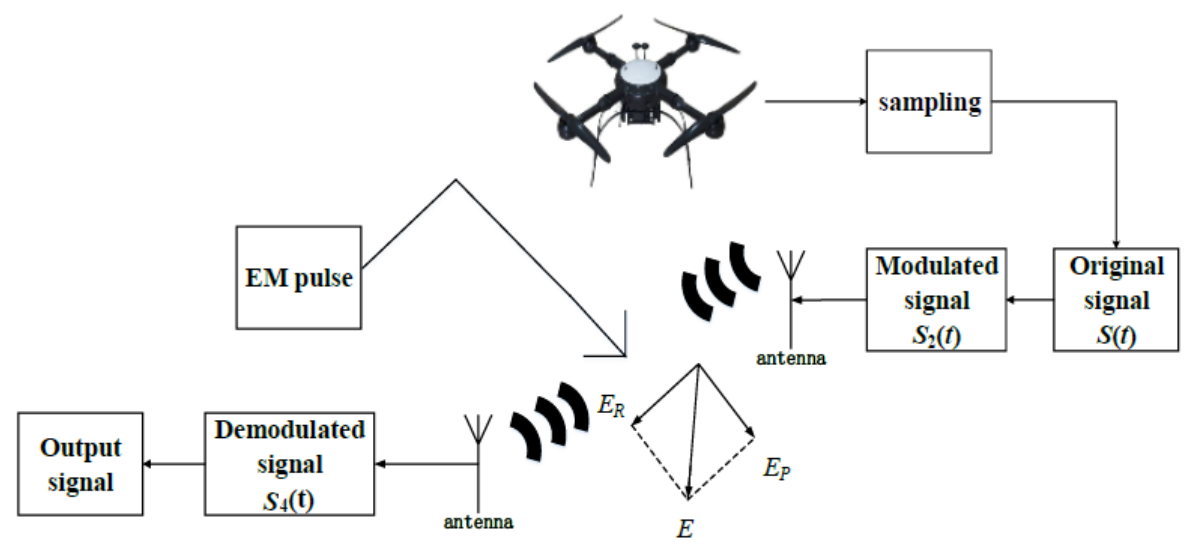

Figure 2. Block diagram of the downlink transmission process

In order to consider the worst case, we superimpose the electromagnetic interference and the modulated signal in the same phase, that is, the formula of the absolute value of the electric field.

$$
|\vec{E}|=\left|\overrightarrow{E_{P}}\right|+\left|\overrightarrow{E_{R}}\right|
$$

In this simulation, we use a baud rate of 4000 , a carrier frequency $f c$ of $4 \mathrm{KHz}$, according to the common transmission anti-noise standard, the sampling frequency $f_{w}$ of the receiving end is $64 \mathrm{KHz}$, the cut-off frequency $f_{k}$ of the low-pass filter is $4 \mathrm{KHz}$, and the error rate $e$ is $5 \%$. The electric field strength $E_{R}$ generated by the communication link at space $(x, y, z)$ is $5 \mathrm{~V} / \mathrm{m}$, as shown in Table 1.

Table 1. Simulation parameters

\begin{tabular}{cccccc}
\hline Baud rate & $\boldsymbol{f}_{\mathrm{c}} / \mathbf{K H z}$ & $\boldsymbol{f}_{\mathrm{w}} / \mathbf{K H z}$ & $\boldsymbol{f}_{\mathrm{k}} / \mathbf{K H z}$ & $\boldsymbol{e} / \%$ & $\boldsymbol{E}_{R} / \mathrm{V} / \mathbf{m}$ \\
\hline 4000 & 4 & 64 & 4 & 5 & 5 \\
\hline
\end{tabular}

\subsection{Influence of High Voltage Transmission Lines on Communication Links}

In real life, especially in remote areas of China, the strong electrical interference generated around the highvoltage transmission line has a great impact on the communication of the UAV, and its electric field expression $E_{p 1}$ is

$$
E_{p_{1}}(t)=c \sin \left(2 \pi f_{D} t+\varphi\right)
$$

Where $c$ is the field strength amplitude, $f_{d}$ is the pulse frequency, and $\varphi$ is the initial phase angle. Superimposing the pulse signal field $E_{P}(t)$ with the modulated signal field $E_{R}(t)$ to interfere with the signal field $E_{N}(t)$

$$
E_{N}(t)=E_{R}(t)+E_{p}(t) .
$$

A sinusoidal pulse with $c$ of $136.70 \mathrm{~V} / \mathrm{m}, f_{d}$ of $50 \mathrm{~Hz}$ and $\varphi$ of 0 rad was used as the EM pulse generated by the high-voltage transmission line, whose waveform is shown in Figure 4(a). The original image Figure 3 was obtained through the demodulation process. The sinusoidal pulse signal was superimposed on the communication link of the UAV, and the interference image was obtained through the demodulation process as shown in Figure 4(b).

Compared with the original image Figure 3, the image in Figure $4(b)$ shows that the character is obviously blurred, the facial features are unclear, the roundness of the picture is significantly weakened, and a large number of stripes appear. At this time, the pulse field strength amplitude is $136.70 \mathrm{~V} / \mathrm{m}$, and the bit error rate is $5.04850 \%$.

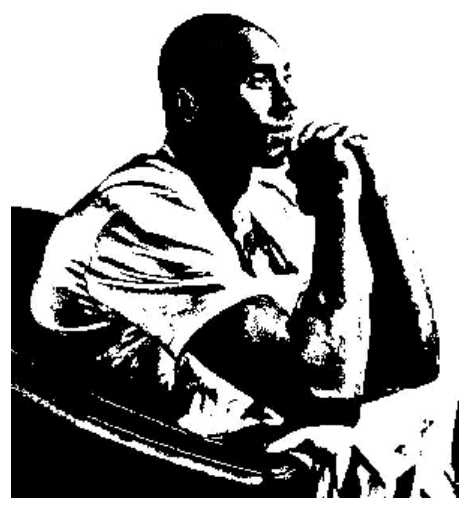

Figure 3. Original image
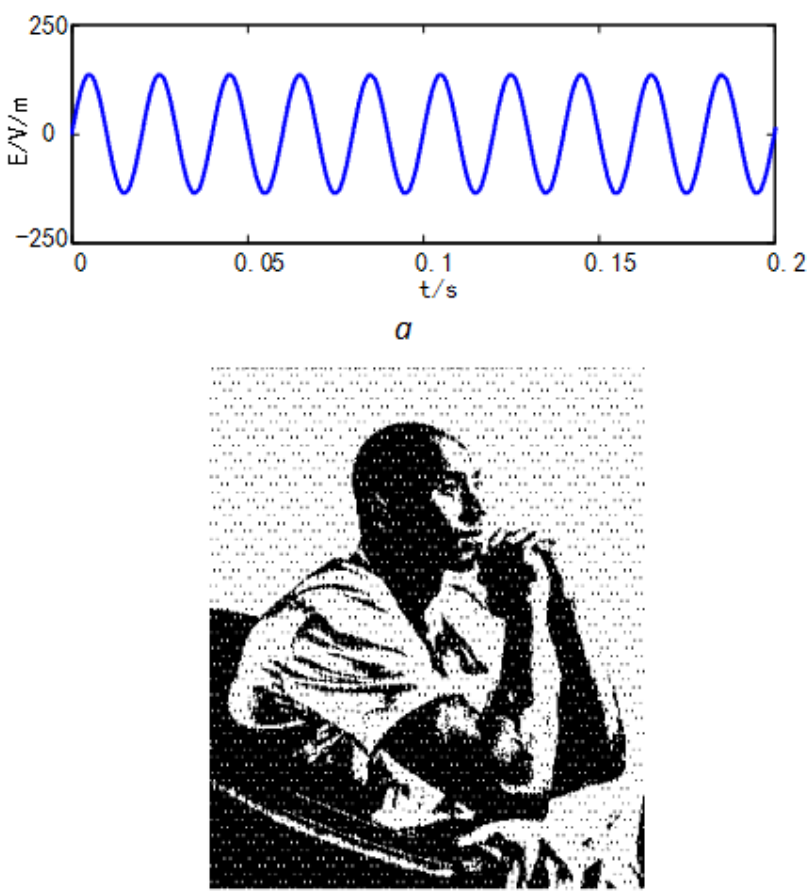

$b$

Figure 4. The influence of UVA communication links with sinusoidal pulse: (a) Sinusoidal pulse waveform, (b) Sinusoidal pulse interference image 


\subsection{Impact of Environmental Noise on Communication Links}

The environmental noise is mainly Gaussian white noise. Gaussian white noise is a kind of noise whose instantaneous value obeys the Gaussian distribution, and its power spectral density is evenly distributed. It is of great significance to analyse the drone in the real environment, whose waveform is shown in Figure 5(a).

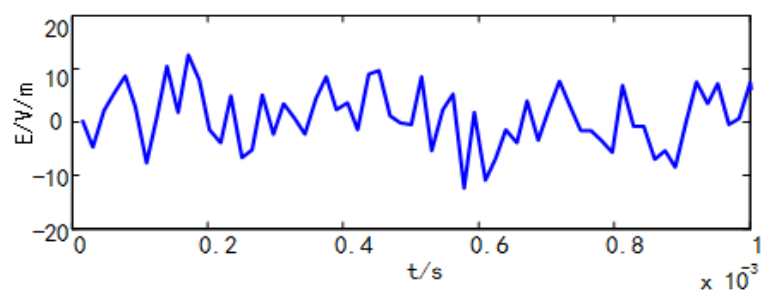

a

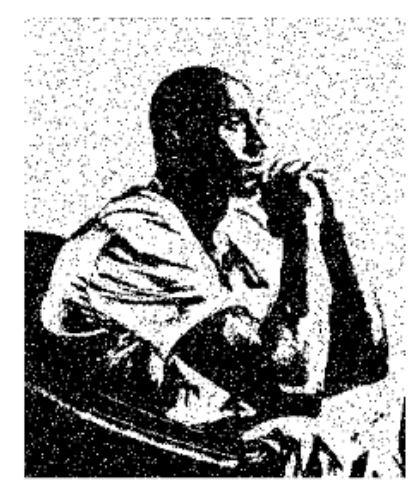

b

Figure 5. The influence of UVA communication links with Gaussian noise: (a) Gaussian noise waveform, (b) Gaussian noise interference image

The Gaussian noise signal is superimposed on the communication link of the UAV, and the interference image is obtained through the demodulation process as shown in Figure 5(b).

Compared with the original image in Figure 3, the image in Figure 5(b) seems to have added salt and pepper noise, and there are many black and white spots, but the overall outline of the character is not greatly disturbed. At this time, the pulse field strength amplitude is $6.29 \mathrm{~V} / \mathrm{m}$ and the bit error rate is $5.00373 \%$.

\subsection{Influence of Electromagnetic Pulse on Communication Link}

\subsubsection{Square Wave Pulse}

Square wave pulse often seriously affects UAV communication in sea environment, etc., and its field analytic function $\mathrm{E}_{\mathrm{p} 2}$ is

$$
E_{P 2}(t)= \begin{cases}c, & 0 \leq t \leq T / 2 \\ 0, & T / 2 \leq t \leq T\end{cases}
$$

Where $T$ is the pulse period.

In this paper, a square wave pulse with $c$ of $3.96 \mathrm{~V} / \mathrm{m}$ and $T$ of $0.25 \mathrm{~ms}$ is applied as an interference pulse to the communication link, whose waveform is shown in Figure 6(a).

The square wave pulse signal is superimposed on the communication link of the UAV, and the interference image is obtained through the demodulation process as shown in Figure 6(b).
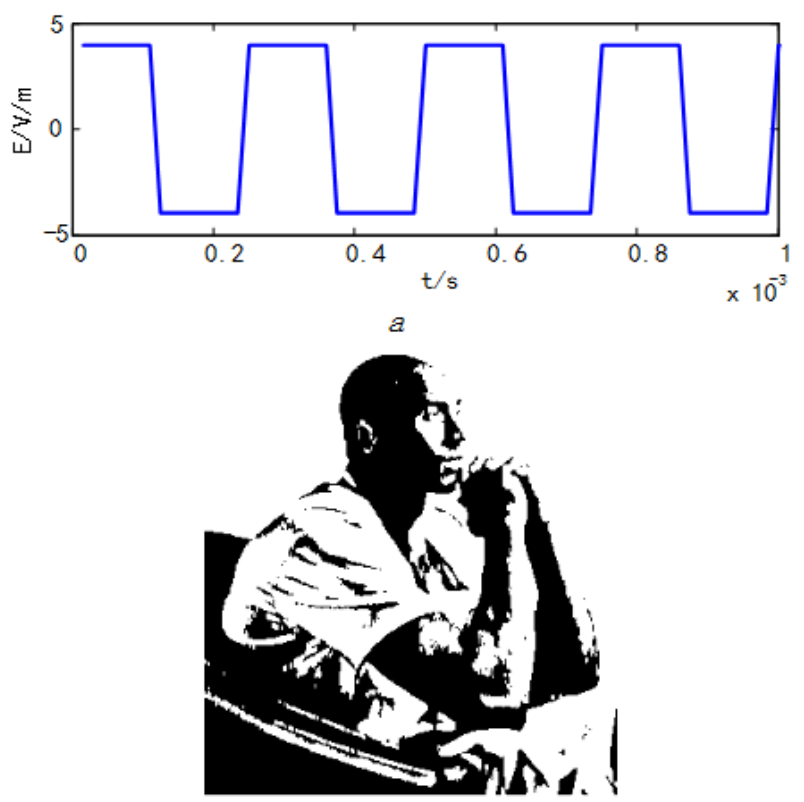

$b$

Figure 6. The influence of UVA communication links with Square wave: (a) Square wave pulse waveform, (b) Square wave interference image

Compared with the original image Figure 3, the face in Figure $6(b)$ is already distorted, and the roundness of the image is also reduced to some extent. At this time, the pulse field strength amplitude is $3.96 \mathrm{~V} / \mathrm{m}$, the bit error rate is $5.05063 \%$.

\subsubsection{Triangle Wave Pulse}

The triangular wave pulse field analytic function $E_{p 3}$ is:

$$
E_{P 3}(t)= \begin{cases}c\left(1+\frac{1}{T / 2}\right) t, & 0 \leq t \leq T / 2 \\ c\left(1-\frac{1}{T / 2}\right) t, & T / 2 \leq t \leq T\end{cases}
$$

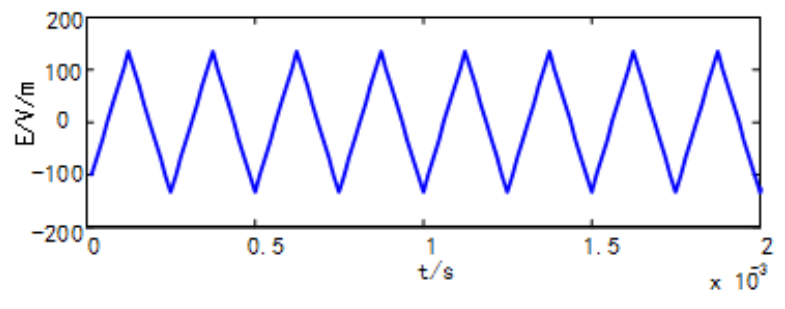

a

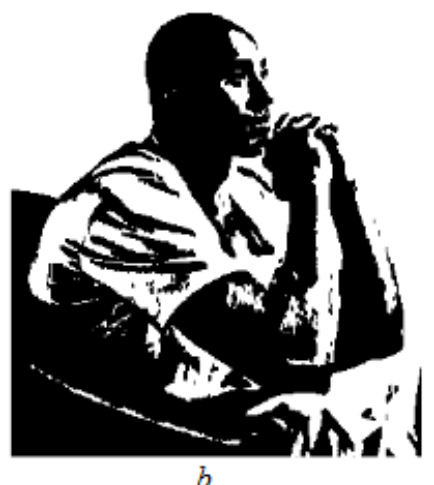

Figure 7. The influence of UVA communication links with Triangle wave: (a) Triangle wave pulse waveform, (b) Triangle wave pulse interference image 
In this paper, a triangular wave pulse with c of 133.59 $\mathrm{V} / \mathrm{m}$ and $T$ of $0.25 \mathrm{~ms}$ is applied as an interference pulse to the communication link, whose waveform is shown in Figure 7(a).

The triangular wave pulse signal is superimposed on the communication link of the UAV, and the interference image is obtained through the demodulation process as shown in Figure 7(b).

Compared with the original image Figure 3, the image in Figure $7(b)$ can be seen that the colour is significantly deeper, and the upper end frame has a part of black lines. At this time, the pulse field strength amplitude is $133.59 \mathrm{~V} / \mathrm{m}$, and the bit error rate is $5.01892 \%$.

\subsubsection{EFT Pulse [20]}

Electrical fast transient (EFT) refers to a limited number of clearly distinguishable pulse sequences or oscillations with limited duration. A single pulse in a pulse group has a specific repetition period, voltage amplitude, rise time, and pulse width. It is an instantaneous high-energy interference that often exists in the power system environment, which has a greater impact on drone communication. It is also called transient pulse disturbance source. It can generate such interference during the switching of power equipment and during lightning discharge. The electric fast transient disturbance group is one of them. The analytic function $E_{p 4}$ is

$$
E_{P 4}(t)=\left\{\begin{array}{cc}
t \times 10^{9}, & 0 \leq t \leq 4.4 \times 10^{-9} \\
4.4 e^{-0.11 \times\left(t-4.4 \times 10^{-9}\right) \times 10^{9}}, & 4.4 \times 10^{-9} \leq t \leq 1 \times 10^{-6}
\end{array}\right.
$$

Based on the Formula (11), the single waveform is shown in Figure 8(a). The EFT single pulse signal is continuously generated to obtain the pulse group, and the EFT pulse group is superimposed on the UAV communication link, and the interference image is obtained through the demodulation process as shown in Figure 8(b).
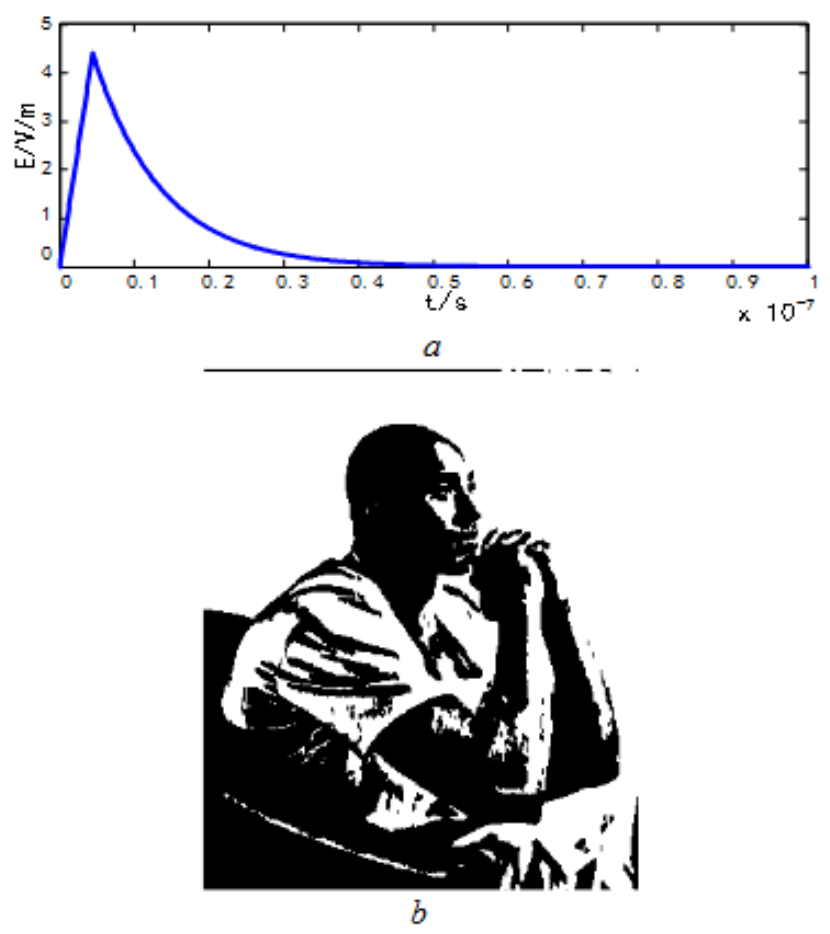

Figure 8. The influence of UVA communication links with EFT pulse: (a) EFT pulse waveform, (b) EFT noise interference image
Compared with the original image Figure 3, the human face in the image in Figure $8(b)$ is not distorted, and the black portion of the entire image is deepened, and the upper edge region has a distinct black border. At this time, the pulse field strength amplitude is $29.18 \mathrm{~V} / \mathrm{m}$, and the error rate of the corresponding image is $5.02265 \%$.

\subsubsection{Surge Pulse [21]}

An electrical surge pulse is a powerful, powerful pulse that is far beyond the normal operating voltage. The instantaneous energy contained in it is enough to cause the drone communication to fail directly. In real life, lightning strikes, start and stop of power equipment, and failure of power supply network operation are easy to cause. Surge pulses may cause loss or damage to the transmitted data or even damage to the equipment. The life of the power equipment is greatly shortened. Even if this ultra-highpower surge pulse is not lightning strike, multiple small surge accumulation effects will cause the semiconductor device. Declining performance, equipment failure, and shortened life. The analytic function $E_{p 5}$ is

$$
\begin{aligned}
& E_{P 5}(t) \\
& =\left\{\begin{array}{cc}
e^{0.481 \times 10^{12} \times t^{2}}-1, & 0 \leq t \leq 1.2 \times 10^{-6} \\
-\left(t-1.2 \times 10^{-6}\right) \times 10^{4}+1, & 1.2 \times 10^{-6} \leq t \leq 1.3 \times 10^{-4} \\
\left(t-1.2 \times 10^{-6}\right) \times 10^{4}-0.424, & 1.3 \times 10^{-4} \leq t \leq 1.58 \times 10^{-4}
\end{array}\right.
\end{aligned}
$$

Based on the formula (12), the single waveform is shown in Figure $9(a)$. The surge pulse signal is continuously applied to the communication link of the drone, and the interference image obtained by the demodulation process is as shown in Figure 9(b).

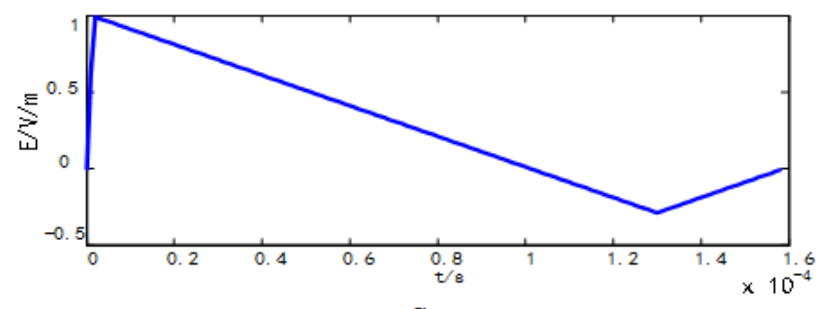

$a$

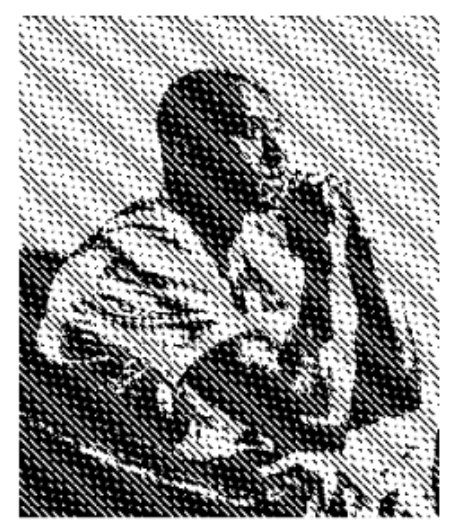

b

Figure 9. The influence of UVA communication links with Surge pulse waveform: (a) Surge pulse waveform, (b) Surge noise interference image

Compared with the original image in Figure 3, there are obvious and numerous cracks with dotted lines of different depths in the image in Figure $9(b)$, and the picture is 
almost completely distorted and unrecognizable. At this time, the pulse amplitude is $10.90 \mathrm{~V} / \mathrm{m}$, and the error rate of the corresponding image is $4.99493 \%$.

\subsection{Comparative Analysis of Different Electromagnetic Interferences}

Combined with the above analysis, the pulse signal amplitude and the bit error rate of different electromagnetic pulse were analysed, as shown in

Table 2. Pulse signal amplitude and bit error rate

\begin{tabular}{lcc}
\hline Noise type & $\boldsymbol{c} / \mathbf{V} / \mathbf{m}$ & $\boldsymbol{e} / \mathbf{o}$ \\
\hline Sinusoidal pulse & 136.70 & 5.01439 \\
Gaussian noise & 6.29 & 5.01306 \\
Square wave pulse & 3.96 & 5.02132 \\
Triangle wave pulse & 133.59 & 4.98640 \\
EFT pulse & 29.18 & 5.02265 \\
Surge pulse & 10.91 & 4.99493 \\
\hline
\end{tabular}

The theoretical simulation results show that the amplitudes of Gaussian noise, square wave pulse and surge pulse are $6.29 \mathrm{~V} / \mathrm{m}$ and $3.96 \mathrm{~V} / \mathrm{m}$, and $10.90 \mathrm{~V} / \mathrm{m}$, when the bit error rate is close to $5 \%$. Due to the low amplitude of electromagnetic pulse, the communication link was influenced seriously with Gaussian noise, square wave pulse and surge pulse.

When the pulse signal interference is detected in the actual operation, a higher protection level is required. The amplitudes of the sinusoidal pulse and the triangular wave pulse are respectively $136.70 \mathrm{~V} / \mathrm{m}$ and $133.59 \mathrm{~V} / \mathrm{m}$, that is, their influence on the communication link is relatively weak, and the required protection level is small in practical use. In addition, the EFT pulse group amplitude is 29.18 $\mathrm{V} / \mathrm{m}$, which requires a moderate degree of protection. On the other hand, in the case where the bit error rate is almost close, the sinusoidal pulse, the Gaussian noise, and the surge pulse have a great influence on the picture, which causes the image to generate cracks or spots of different degrees. Especially surge pulses, which make the image Almost unrecognizable, need to be most protected. The square wave pulse will make the image lighter and blur, and the triangular wave pulse and EFT pulse group will make the image darker and the resolution will be lower, therefore, higher level of protection is required when more accurate picture information is required.

\section{Conclusion}

By analysing the principle of BPSK modulation and demodulation, a typical electromagnetic pulse model including sinusoidal pulse, square wave pulse, triangular wave pulse, Gaussian noise, electrical fast transient pulse group (EFT) and surge pulse has been established. For gaussian pulse, triangular pulse, EFT pulse and surge pulse, which have a greater impact on the communication link, require significant protection. The analysis of their impact of UAV's downlink communication link and bit error rate provides theoretical basis for classification of electromagnetic pulses, methods of anti-interference suppression, and design optimization of UAV.

\section{Acknowledgements}

This paper is supported by the National Natural Science Foundation of China (51475246); Natural Science Foundation of Jiangsu Province (BK20161019); Aviation Science Fund (20172552017).

\section{References}

[1] Eschmann, C., Kuo, C., Kuo, C., et al.: 'Unmanned aircraft systems for remote building inspection and monitoring'. 6th European Workshop on Structural Health Monitoring, Dresden, Germany, 2012, pp.1-8.

[2] Jordan, Sophie., Moore, Julian., Hovet, Sierra., et al.: 'State-of-theart technologies for UAV inspections', IET Radar, Sonar \&amp; Navigation,2018, 12, (2), pp.151-164.

[3] Nicol, S.E., Walton, G., Westbrook, L.D., et al.: 'Future satellite communications to military aircraft', Electronics \&amp; Communication Engineering Journal, 2000, 12, (1), pp. 15-26.

[4] P. B. Charlesworth.: 'Simulating Missions of a UAV with a Communications Payload', 2013 UKSim 15th International Conference on Computer Modelling and Simulation, Cambridge, 2013, pp. 650-655.

[5] A. Tato., C. Mosquera., I. Gomez.: 'Link adaptation in mobile satellite links: field trials results', 2016 8th Advanced Satellite Multimedia Systems Conference and the 14th Signal Processing for Space Communications Workshop (ASMS/SPSC), Palma de Mallorca, 2016, pp. 1-8.

[6] Chen, Chen., Wei, Yan., Yang, Zhao., et al.: 'Simulation and analysis of EMP transient electromagnetic effect of aircraft', The Journal of Engineering, Impress.

[7] M. R. Cabello., et al.: 'SIVA UAV: A Case Study for the EMC Analysis of Composite Air Vehicles', IEEE Transactions on Electromagnetic Compatibility, 2017, 59, 4, pp. 1103-1113.

[8] AndreadouN.Nivoi PavlidouF.: 'PLC hannel: impulsive noise modeling and its performance evaluation under different array coding schemes', IEEE Trans. Power Deliv., 2009, 24, (2), pp. 585-595.

[9] Rahman, Munshi Mahbubur., Majumder, Satya Prasad.: 'Analytical bit error rate performance evaluation of an orthogonal frequency division multiplexing power line communication system impaired by impulsive and Gaussian channel noise', The Journal of Engineering, 2015, (2), pp. 71-77.

[10] Shuxia Guo., Yafeng Wang., Ruibing Liu., et al.: 'Multidimensional and complicated electromagnetic interference hardware-in-the-loop simulation method', Journal of Systems Engineering and Electronics, 2015, 26(6): 1142-1148.

[11] Jacob D., Vignesh R., Chuck B.: 'Performance test of unmanned aerial systems communication links in a severe multipath environment', 2016 IEEE International Symposium on Electromagnetic Compatibility (EMC), 2016: 862-867.

[12] Pienaar H., Andriambeloson J.A., Davidson D.B.:'RF shielding for multi-copter based electromagnetic metrology platforms', 2016 IEEE Conference on Antenna Measurements\& Applications (CAMA), 2016: 1-4.

[13] Devender., K. Nageswara Rao., K. Suryanarayana., et al.: 'Electromagnetic interference (EMI) suppression techniques - a case study', Proceedings of the International Conference on Electromagnetic Interference and Compatibility (IEEE Cat. No.02TH8620), Bangalore, India, 2002, pp. 221-225.

[14] Yu, Zhanqing., Wang, Xiaorui., Zeng, Rong., et al.: 'Analysis of factors influencing the parameters of electromagnetic environment', The Journal of Engineering, Impress.

[15] W. Ting., S. Qijun., G. Yougang.:'The Analysis about UWB's EMI Cause to UAV System Based on Matlab/Simulink', The 2006 4th Asia-Pacific Conference on Environmental Electromagnetics, Dalian, 2006, pp. 795-797.

[16] Zhao Yang., See Kye Yak., Li Shijin., et al.: 'Fast diagnosis, prediction and signal characterization of radiated electromagnetic interference (EMI) noise', 2008 International Conference on Microwave and Millimeter Wave Technology, Nanjing, 2008, pp. 1446-1449. 
[17] J. Kelin., W. Jingmei., W. Chang., et al.: 'A New Method for Conducted EMI Noise Diagnosis', 2007 8th International Conference on Electronic Measurement and Instruments, Xi'an, 2007, pp. 4-59-4-63.

[18] S. Q. Hadi., P. Ehkan., M. S. Anuar., et al.: 'Performance comparison of STBC-FT based OFDM wireless communication system using M-QAM and M-PSK modulation techniques', 2016 3rd International Conference on Electronic Design (ICED), Phuket, 2016, pp. 174-179.
[19] S. O. Popescu., G. Budura., A. S. Gontean.: 'Review of PSK and QAM - Digital modulation techniques on FPGA', 2010 International Joint Conference on Computational Cybernetics and Technical Informatics, Timisoara, 2010, pp. 327-332.

[20] IEC61000-4-4: '2012 Electromagnetic compatibility (EMC) Part-4-4: Testing and measurement techniques-Electrical fast transient/burst immunity test'.2012

[21] IEC61000-4-5: '2014 Electromagnetic compatibility (EMC) Part-4-5: Testing and measurement techniques-Surge immunity test'. 2014. 